\title{
Fully Self-Consistent $N$-body Simulation of Star Cluster in the Galactic Center
}

\author{
M. Fujii ${ }^{1,3}$, M. Iwasawa ${ }^{2,3}$, Y. Funato ${ }^{2}$ and J. Makino ${ }^{3}$ \\ ${ }^{1}$ Department of Astronomy, Graduate School of Science, The University of Tokyo, \\ 7-3-1 Hongo, Bunkyo, Tokyo 113-0033 \\ email: fujii@cfca.jp \\ ${ }^{2}$ Department of General System Studies, Graduate School of Arts and Sciences, \\ The University of Tokyo, 3-8-1 Komaba, Meguro, Tokyo 153-8902 \\ email: iwasawa@cfca.jp, funato@artcompsci.org \\ ${ }^{3}$ Division of Theoretical Astronomy, National Astronomical Observatory of Japan, \\ 2-21-1 Osawa, Mitaka, Tokyo, 181-8588 \\ email: makino@cfca.jp
}

\begin{abstract}
We have developed a new tree-direct hybrid algorithm, "Bridge". It can simulate small scale systems embedded within large- $N$ systems fully self-consistently. Using this algorithm, we have performed full $N$-body simulations of star clusters near the Galactic center (GC) and compared the orbital evolutions of the star cluster with those obtained by "traditional" simulations, in which the orbital evolution of the star clusters is calculated from the dynamical friction formula. We found that the inspiral timescale of the star cluster is shorter than that obtained with traditional simulations. Moreover, we investigated the eccentricities of particles escaped from the star cluster. Eccentric orbit of the star cluster can naturally explain the high eccentricities of the observed stars.
\end{abstract}

Keywords. galaxies: star clusters, methods: n-body simulations, Galaxy: center, kinematics and dynamics, stellar dynamics

A few dozens of very young and massive stars have been found in the central parsec of the Galaxy (Krabbe et al. 1995; Paumard et al. 2006). Some of these stars have high eccentricities (Paumard et al. 2006; Lu et al. 2006). In the central parsec, in situ formation of these stars seems difficult because of the strong tidal field of the central black hole (BH). Star cluster inspiral scenario (Gerhard 2001) can overcome this difficulty, but numerical simulations have shown that it would take too long time for inspiraling (Portegies Zwart et al. 2003; Gürkan \& Rasio 2005). In these works the orbit of the cluster was calculated using the dynamical friction formula (Chandrasekhar 1943). This "traditional" analytic approach might have overestimated the inspiral timescale. Fujii, Funato \& Makino (2006) showed that the orbital decay of the satellite is much faster than those calculated analytically. This difference was caused by particles escaped from the satellite. It should also occur in the case of star clusters. Therefore, a fully self-consistent $N$-body simulation is necessary.

However, such a fully self-consistent $N$-body simulation has been impossible with conventional numerical methods. While star clusters need a very accurate scheme, galaxies contain too many particles to use it. To overcome this problem, we have developed a new tree-direct hybrid scheme, the "Bridge" scheme (Fujii et al. 2007a). In this scheme, the internal interactions of star clusters are calculated accurately using the direct Hermite scheme, while all other interactions are calculated with the tree algorithm (Barnes \& Hut 1986). We combined these two methods by extending the idea of the MVS scheme (Wisdom \& Holman 1991; Kinoshita, Yoshida, \& Nakai 1991). 

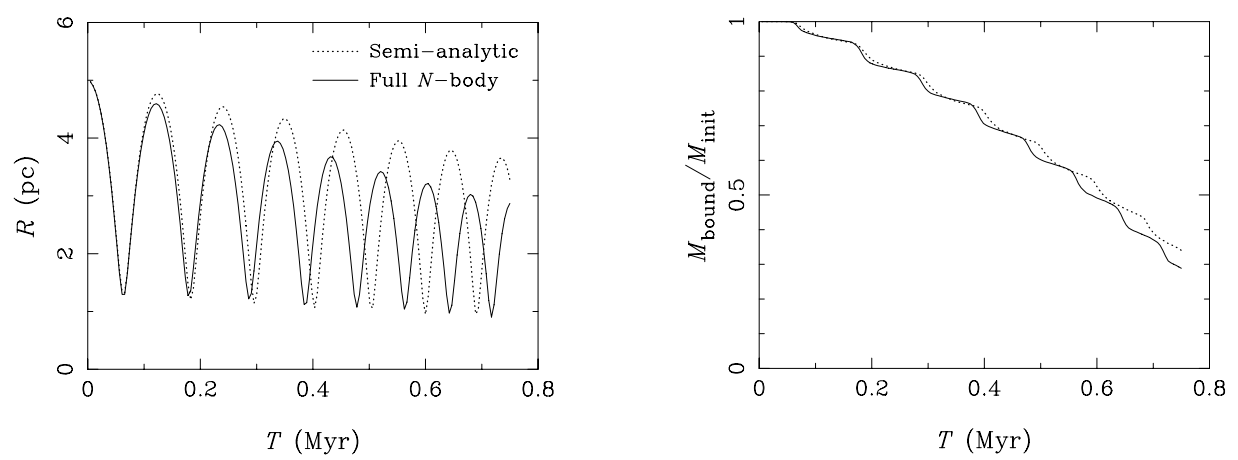

Figure 1. The distance of the star cluster from the GC (left) and the bound mass of the star cluster (right) plotted as a function of time. Solid and dotted curves show the result of the full $N$-body simulation and that of the "traditional" simulations, respectively. The initial orbit of the star cluster is eccentric

Using the Bridge scheme, we performed fully self-consistent $N$-body simulations of star clusters within their parent galaxies and compared the results with those obtained using the "traditional" simulations (Fujii et al. 2007b). We adopted a King model as a model of the star cluster and assigned each star a mass randomly drawn from a Salpeter (1955) initial mass function. We calculated two orbits; one is circular with the initial position from the GC, $r_{\text {init }}$, is $2 \mathrm{pc}$ and the other is eccentric with $r_{\text {init }}=5$ pc. We used GRAPE- 6 (Makino et al. 2003) for force calculation.

Fig. 1 shows the orbit and bound-mass of the star cluster with eccentric orbit. The orbital decay in the full $N$-body simulation is faster by $30-40 \%$ than that in traditional simulation. On the other hand, the evolution of the bound mass of the star cluster is almost the same between the two. This result suggests that previous studies underestimated the inspiral timescale of star clusters. In addition, eccentric orbits are preferable,because star clusters with eccentric orbits can approach to the GC much faster than those with circular orbits. We also investigated the eccentricities, $e$, of stars escaped from the star cluster (i.e. unbound stars). In the case of the eccentric orbit, the eccentricities of the escapers are higher than those in the case of the circular orbit. Thus, if the star cluster is initially in an eccentric orbit, it naturally explains the observations.

\section{References}

Barnes, J. \& Hut, P. 1986, Nature 324, 446

Chandrasekhar, S. 1943, ApJ 97, 255

Fujii, M., Funato, Y., \& Makino, J. 2006. PASJ 58, 743

Fujii, M., Iwasawa, M., Funato, Y., \& Makino, J. 2007a, PASJ accepted, arXiv: 0706.2059

Fujii, M., Iwasawa, M., Funato, Y., \& Makino, J. 2007b, arXiv: 0708.3719

Gerhard, O. 2001, ApJ 546, L39

Gürkan, M. A. \& Rasio, F. A. 2005, ApJ 628, 236

Kinoshita, H., Yoshida, H. \& Nakai, H. 1991, Cel. Mech. and Dyn. Astr. 50, 59

Krabbe, A. et al. 1995, ApJ 447, L95

Lu, J.R. et al. 2006, JPhCS 54, 279

Makino, J., Fukushige, T., Koga, M., \& Namura, K. 2003, PASJ 55, 1163

Paumard, T. et al. 2006 ApJ 643, 1011

Portegies Zwart, S. F., McMillan, S. L. W., \& Gerhard, O. 2003, ApJ 593, 352

Salpeter, E. E. 1955, ApJ 121, 161

Wisdom, J. \& Holman, M. 1991, AJ 102, 152 